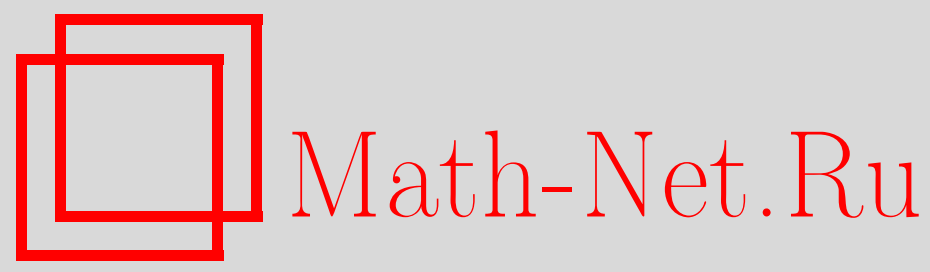

А. В. Дмитрук, Н. В. Кузькина, Теорема существования в задаче оптимального управления на бесконечном интервале времени, Матем. заметки, 2005, том 78, выпуск 4, 503518

DOI: https://doi.org/10.4213/mzm2609

Использование Общероссийского математического портала Math-Net.Ru подразумевает, что вы прочитали и согласны с пользовательским соглашением http://www.mathnet.ru/rus/agreement

Параметры загрузки:

IP: 35.173 .219 .12

26 апреля 2023 г., 15:43:49

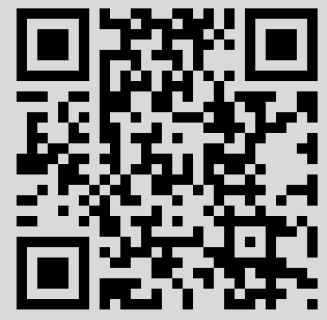




\title{
ТЕОРЕМА СУЩЕСТВОВАНИЯ В ЗАДАЧЕ ОПТИМАЛЬНОГО УПРАВЛЕНИЯ НА БЕСКОНЕЧНОМ ИНТЕРВАЛЕ ВРЕМЕНИ
}

\author{
А. В. Дмитрук, Н.В. Кузькина
}

\begin{abstract}
Рассматривается задача оптимального управления на бесконечном интервале времени. Система линейна по управлению, функционал выпуклый по управлению, а множество управлений есть выпукльй компакт. Относительно поведения функционала на бесконечности предлагается новое условие, которое слабее уже известных. При его выполнении доказана теорема существования решения. Рассмотрены некоторые частные случаи и предложена общая абстрактная схема.

Библиографория: 13 названий.
\end{abstract}

1. Введение. Задачи оптимального управления на бесконечном интервале времени возникают как в теоретических, так и в прикладных разделах математики, например, в динамических моделях математической экономики [1]-[7]. Вопрос о существовании решения в таких задачах решается не столь просто, как в задачах на конечном отрезке времени [8]-[10]. В имеющихся на эту тему работах условия существования либо чересчур ограничительны, либо сложно формулируются и поэтому не очень просто проверяются. В данной работе мы рассматриваем широкий класс задач, включающий, в частности, многие задачи экономической динамики, и предлагаем довольно естественные условия, обеспечивающие существование решения в этих задачах, которые слабее всех известных условий.

2. Постановка задачи, предположения. На полуинтервале $[0, \infty)$ будем рассматривать $n$-мерные функции $x(t)$, абсолютно непрерьвные на любом отрезке [0,T] (будем писать, что $x(\cdot) \in A C[0, \infty)$ ), и измеримые $r$-мерные функции $u(t)$, которые на любом отрезке $[0, T]$ существенно ограничены; пространство таких функций обозначим $L_{\infty}[0, \infty)$.

Обратим внимание, что функции из $A C[0, \infty)$ могут не быть абсолютно непрерывными на всей полупрямой $[0, \infty)$. Аналогичное замечание относится и к функциям из $L_{\infty}[0, \infty)$. (Более специальных обозначений для пространств $A C$ и $L_{\infty}$ на $[0, \infty)$ мы не вводим, так как эти пространства будут у нас пониматься только в указанном смысле.)

Работа выполнена при частичной поддержке Российского фонда фундаментальных исследований, грант № 04-01-00482, и программы "Ведущие научные школы", грант № НШ-304.2003.1. 
На пространстве пар функций $(x, u)$ будем рассматривать следующую задачу оптимального управления:

$$
\begin{gathered}
J(x, u)=\beta(x(0))+\int_{0}^{\infty} \varphi(t, x, u) d t \rightarrow \min , \\
\dot{x}=f(t, x, u), \\
x(0) \in M_{0}, \\
u(t) \in U(t, x(t)), \\
x(t) \in S(t) .
\end{gathered}
$$

Пары функций $(x, u) \in A C \times L_{\infty}[0, \infty)$, удовлетворяющие на $[0, \infty)$ ограничениям (2)-(5), будем называть допустимыми (как обычно, для измеримых функций все соотношения понимаются вьполненными почти всюду), а множество всех допустимых пар обозначим через $\Omega$. Функционал (1) рассматривается на всех парах $(x, u) \in \Omega$, для которых соответствующий интеграл Лебега по любому отрезку $[0, T]$ существует и при $T \rightarrow \infty$ сходится к конечному или бесконечному пределу. Таким образом,

$$
J(x, u)=\lim _{T \rightarrow \infty} J_{T}(x, u), \quad \text { где } \quad J_{T}(x, u)=\beta(x(0))+\int_{0}^{T} \varphi(t, x, u) d t
$$

а предел мы понимаем в указанном расширенном смысле. (Ниже мы приведем условие, гарантируюшее сходимость интеграла на любой паре $(x, u) \in \Omega$.)

ЗАмЕЧАнИЕ. Чтобы избавиться от “неудобного” вопроса о сходимости интеграла в (1), в некоторых работах предлагается изменить само понятие оптимальности, а точнее, сам принцип сравнения двух допустимых пар: предлагается сравнивать не предельные значения функционала (1) (которых может не существовать), а рассматривать поведение разности функционалов $J_{T}\left(x^{\prime \prime}, u^{\prime \prime}\right)-J_{T}\left(x^{\prime}, u^{\prime}\right)$ на отрезках $[0, T]$ при $T \rightarrow \infty$. При этом естественного однозначного толкования, для какой пары семейство значений $J_{T}$ “лучше", не получается, приходится рассматривать несколько различных толкований, и соответственно, несколько разных понятий оптимальности (см. работы [4]-[7] и литературу в них). В данной статье мы не касаемся этих обобщений. Допустимые пары сравниваются здесь обычным образом - по значению функционала на них, и соответственно, оптимальность понимается как достижение минимально возможного значения функционала.

ПРЕдПолоЖЕния. Будем считать, что вьполнены следующие условия.

П1. Функция $f(t, x, u)$ непрерывна по паре $(t, x)$ и линейна по $u$, т.е.

$$
f(t, x, u)=a(t, x)+B(t, x) u,
$$

где $n$-мерный вектор $a$ и $(n \times r)$-матрица $B$ непрерьвно зависят от $(t, x)$.

П2. Множество $S(t)$ замкнуто для всех $t \geqslant 0$, а зависимость его от $t$ произвольна.

П3. Множество $M_{0}$ есть компакт в $\mathbb{R}^{n}$.

П4. Многозначное отображение $U: \mathbb{R}^{1+n} \rightarrow \mathbb{R}^{r}$ имеет замкнутый график, и для любых $(t, x)$ множество $U(t, x)$ есть выпукльй компакт. 
П5. Функция $f$ вместе с отображениями $S$ и $U$ удовлетворяет условию Филиппова [8], т.е. существует такое $c$, что для всех $t \geqslant 0, x \in S(t), u \in U(t, x)$

$$
(x, f(t, x, u)) \leqslant c\left(|x|^{2}+1\right) .
$$

П6. Функция $\beta(\cdot)$ непрерьвна на $M_{0}$.

П7. Функция $\varphi(t, x, u)$ непрерьвна по $(t, x, u)$ и вьпукла по $u$.

Обратим внимание, что если $S(t)$ и $U(t, x)$ равномерно ограничены, то предположение П5 заведомо вьполнено. Однако выполнение П5 и в этом, и в общем случае еще не гарантирует сушествования допустимой траектории системы (2)-(5) на заданном отрезке $[0, T]$, и тем более на $[0, \infty)$. Наличие допустимой траектории, как и всегда в теоремах существования в задачах на экстремум, будет не доказываться, а просто постулироваться.

Все эти предположения вполне стандартны для теорем существования в задаче на фиксированном конечном отрезке. Кроме них, мы примем еще одно предположение относительно поведения семейства функций $\varphi(t, x(t), u(t))$ на бесконечности.

Для любого числа $a$ обозначим $a^{+}=\max (a, 0), a^{-}=\max (-a, 0)$ (обе эти величины неотрицательные), так что $a=a^{+}-a^{-}$.

Назовем куском функционала $J$ на отрезке $\left[T^{\prime}, T^{\prime \prime}\right]$ величину

$$
\int_{T^{\prime}}^{T^{\prime \prime}} \varphi(t, x(t), u(t)) d t
$$

Следующее предположение является ключевым для наших целей.

ПРЕДПОЛОЖЕНИЕ П8. Отрицательные части кусков функционала сходятся к 0 при $T^{\prime}, T^{\prime \prime} \rightarrow \infty, T^{\prime}<T^{\prime \prime}$, равномерно по всем допустимьм траекториям.

Другими словами, для любого $\varepsilon>0$ существует $T_{\varepsilon}$ такое, что для всех $T^{\prime \prime}>T^{\prime}>T_{\varepsilon}$, для всех $(x, u) \in \Omega$

$$
\int_{T^{\prime}}^{T^{\prime \prime}} \varphi(t, x(t), u(t)) d t>-\varepsilon .
$$

Это условие очевидно эквивалентно следующему: существует функция $\alpha(T) \rightarrow 0+$ при $T \rightarrow \infty$ и число $T_{0}$ такие, что для любого $T>T_{0}$ и для любых $T^{\prime \prime}>T^{\prime} \geqslant T$, $(x, u) \in \Omega$

$$
\int_{T^{\prime}}^{T^{\prime \prime}} \varphi(t, x(t), u(t)) d t \geqslant-\alpha(T) .
$$

Ниже мы укажем некоторые случаи, когда это предположение заведомо выполнено.

Установим, что вьполнение предположения П8 на $\Omega$ гарантирует сходимость интеграла (в указанном выше расширенном смысле) для любой пары $(x, u) \in \Omega$. Нам потребуется следующий простой факт.

Лемма 1. Пусть числовая последовательность $\gamma_{k}$ не имеет предела (конечного или бесконечного). Тогда существуют числа $z_{1}<z_{2}$ такие, что для любого номера $K$ найдутся $k_{1}, k_{2}>K$, для которых $\gamma_{k_{1}}<z_{1} u \gamma_{k_{2}}>z_{2}$. 
ДокАЗАтЕльство. Так как последовательность $\gamma_{k}$ не имеет предела, из нее можно выделить две подпоследовательности, сходяшиеся к разным пределам: $\gamma_{k_{s}^{1}} \rightarrow c_{1}$ и $\gamma_{k_{s}^{2}} \rightarrow c_{2}$, где $c_{1}<c_{2}$ (при этом возможны случаи $c_{1}=-\infty, c_{2}=+\infty$ ). Возьмем любые $z_{1}, z_{2} \in \mathbb{R}$ такие, что $c_{1}<z_{1}<z_{2}<c_{2}$. Тогда для любого номера $K$ найдутся номер $k_{1}>K$ из последовательности $k_{s}^{1}$ и номер $k_{2}>K$ из последовательности $k_{s}^{2}$ такие, что $\gamma_{k_{1}}<z_{1}, \gamma_{k_{2}}>z_{2}$. Лемма доказана.

ЛЕмма 2. Пусть функиионал $Ј$ удовлетворяет предположению П8. Тогда для всех $(x, u) \in \Omega$ соответствуюший интеграл сходится либо к конечному пределу, либо $\kappa+\infty$.

ДокАЗАТЕльСтво. Допустим сначала, что для некоторой пары $(x, u) \in \Omega$ интеграл не сходится (в нашем расширенном смысле), т.е. не существует

$$
\lim _{T \rightarrow \infty} \int_{0}^{T} \varphi(t, x(t), u(t)) d t
$$

Значит, этот предел не существует и для некоторой счетной последовательности $T \rightarrow \infty$. Тогда по лемме 1 найдутся числа $z_{1}>z_{2}$ такие, что для любого $T$ существуют $T_{1}>T$ и $T_{2}>T_{1}$ изэтой счетной последовательности, для которых $J_{T_{1}}(x, u)>z_{1}, J_{T_{2}}(x, u)<z_{2}$, и поэтому

$$
\int_{T_{1}}^{T_{2}} \varphi(t, x(t), u(t)) d t=J_{T_{2}}(x, u)-J_{T_{1}}(x, u)<z_{2}-z_{1}=\text { const }<0 .
$$

Но это противоречит предположению П8. Значит, наше допущение неверно.

Рассмотрим теперь случай, когда предел (7) равен $-\infty$. Тогда для любого $T^{\prime}$ найдется такое $T^{\prime \prime}>T^{\prime}$, что

$$
\int_{0}^{T^{\prime \prime}} \varphi(t, x(t), u(t)) d t<\int_{0}^{T^{\prime}} \varphi(t, x(t), u(t)) d t-1
$$

и, следовательно,

$$
\int_{T^{\prime}}^{T^{\prime \prime}} \varphi(t, x(t), u(t)) d t<-1
$$

т.е. при $\varepsilon=1$ опять приходим к противоречию с П8. Лемма доказана.

Таким образом, для любой допустимой пары $(x, u)$ величина

$$
J(x, u)=\lim _{T} J_{T}(x, u)
$$

определена корректно, причем $J$ всег да принимает либо конечные значения, либо $+\infty$. Кроме того, из доказательства леммы 2 видно, что П8 является наиболее естественным предположением, обеспечивающим эти свойства функционала $J$.

ТЕОРемА 1 (основная). Пусть при сделанных предположсениях П1-П8 существует хотя бъ одна пара $(x, u) \in \Omega$, на которой $J(x, u)<+\infty$. Тогда найдется пара $\left(x_{0}, u_{0}\right) \in \Omega$, на которой функционал достигает своего минимального значения (т.е. в поставленной задаче существует решение).

Для доказательства нам потребуется ряд свойств функционала $J$ и множества допустимых траекторий на фиксированном отрезке $[0, T]$. 


\section{3. Некоторые свойства для фиксированного отрезка.}

ЛЕмма 3. Пусть $U(y)$ - полунепрерывное сверху многозначное отображсение $\mathbb{R}^{m} \rightarrow \mathbb{R}^{r}$ с компактными значениями. Тогда на любом компактном (а значит, и на любом ограниченном) множестве у значения $U(y)$ равномерно ограничены, m.е. для любого компакта $K \subset \mathbb{R}^{m}$ существует такая константа $R$, что для любого у $\in K$ мнохество $U(y)$ содержится в шаре $B_{R}(0)$.

ДокАЗАТЕЛЬСТвО. В силу полунепрерьвности сверху отображения $U(y)$ для любого $y$ существует некоторая его окрестность $\mathscr{O}(y)$ такая, что для любого $y^{\prime} \in O(y)$ вьполнено включение $U\left(y^{\prime}\right) \subset U(y)+B_{1}(0)$. Объединение окрестностей $\mathscr{O}(y)$ по всем $y \in K$ покрывает весь компакт $K$, и по определению компакта из этого покрытия можно выбрать конечное подпокрытие. То есть существует конечное число точек $y_{1}, \ldots, y_{m} \in K$ и их окрестностей $\mathscr{O}\left(y_{i}\right)$ таких, что для любого $y^{\prime} \in \mathscr{O}\left(y_{i}\right)$ множество $U\left(y^{\prime}\right)$ содержится в $U\left(y_{i}\right)+B_{1}(0)$ и эти окрестности покрьвают весь компакт $K$.

Объединение $V$ ограниченных множеств $U\left(y_{i}\right)+B_{1}(0)$ по всем $i=1, \ldots, m$ также будет ограниченным, т.е. будет целиком содержаться в шаре $B_{R}(0)$ при некотором $R$. А так как для любого $y \in K$ найдется номер $i$, для которого $y \in \mathscr{O}\left(y_{i}\right)$, то $U(y) \subset$ $U\left(y_{i}\right)+B_{1}(0) \subset V$, и поэтому $U(y) \subset B_{R}(0)$.

СлЕДСТВИЕ. Пусть отображсение $U(t, x): \mathbb{R}^{1+n} \rightarrow \mathbb{R}^{r}$ имеет замкнутый график и компактные значения. Тогда для любого $T>0$ и любого ограниченного множества $Q \subset \mathbb{R}^{n}$ найдется такое $R=R(T, Q)$, что для любого $t \in[0, T]$, для любого $x \in Q$ выполнено включение $U(t, x) \subset B_{R}(0)$.

ДокАЗАТЕЛЬСТВО получается применением леммы 3 к отображению $U(y)$, где $y=$ $(t, x)$, и компакту $K=[0, T] \times \bar{Q}$. При этом надо учесть, что замкнутость графика этого отображения эквивалентна его полунепрерьвности сверху.

Лемма 4. Пусть $U(t, x)$ имеет замкнутый график и компактные значения, $и$ пусть функиия $f(t, x, u)$ вместе с отображсениям $S$ и $U$ удовлетворяет предположению П5 (т.е. условию Филиппова). Пусть также дано ограниченное мнохество $M_{0} \subset \mathbb{R}^{n}$. Тогда для любого $T$ существуют такие константы $D_{T}, D_{T}^{\prime}, R_{T}$, что для любого решения системы (2)-(5) почти всюду на $[0, T]$ выполняются очен$\kappa u$

$$
|x(t)| \leqslant D_{T}, \quad|\dot{x}(t)| \leqslant D_{T}^{\prime}, \quad|u(t)| \leqslant R_{T} .
$$

ДокАЗАтЕльСтво. Рассмотрим функцию $z(t)=|x(t)|^{2}+1$. Для нее на траекториях системы $(2)$ имеем $\dot{z}=2(x, f)$, поэтому в силу П5 имеем $\dot{z} \leqslant 2 c\left(|x|^{2}+1\right)$, т.е. $\dot{z} \leqslant 2 c z$. Учитьвая, что $z(t) \geqslant 0$, отсюда получаем, что для любого $t \geqslant 0$ вьполнено неравенство $z(t) \leqslant z(0) e^{2 c t}$. Если $\left|M_{0}\right| \leqslant r$, то на отрезке $[0, T]$ будет вьполнено неравенство $z(t) \leqslant\left(r^{2}+1\right) e^{2 c T}$, из которого и вытекает первая требуемая оценка в (8).

Так как $|x(t)| \leqslant D_{T}$, то по следствию из леммы $3|u(t)| \leqslant R_{T}$ с некоторой $R_{T}$, поэтому тройка $(t, x(t), u(t))$ всегда принадлежит компакту $[0, T] \times B_{D_{T}}(0) \times B_{R_{T}}(0)$. В силу непрерьвности функции $f$ она ограничена на этом компакте некоторой константой $D_{T}^{\prime}$. Отсюда $|\dot{x}(t)| \leqslant D_{T}^{\prime}$. Лемма доказана.

Таким образом, множество решений $x(t)$ системы (2)-(5) равномерно ограничено и равномерно липшищево, а множество управлений $u(t)$ равномерно ограничено. Отсюда в силу теорем Асколи-Арцела и Алаоглу [11], получаем 
СлЕДСТВИЕ. Множество решений $x(t)$ системы (2)-(5) предкомпактно в пространстве $C[0, T]$, а соответствующее множсество управлений и $(t)$ предкомпактно в пространстве $L_{\infty}[0, T]$ относительно *-слабой топологии (т.е. относительно топологии, определяемой функиионалами из $\left.L_{1}[0, T]\right)$.

Пусть $\Omega_{T}$ есть множество всех $(x, u) \in A C[0, T] \times L_{\infty}[0, T]$, удовлетворяющих на $[0, T]$ ограничениям (2)-(5). Из только что полученного следствия вытекает, что $\Omega_{T}$ предкомпактно относительно произведения равномерной и $*$-слабой топологий. Как известно, на ограниченных множествах в пространстве $L_{\infty}[0, T]$ *-слабая топология метризуема (так как $L_{1}$ сепарабельно), поэтому при ее изучении достаточно рассматривать сходящиеся последовательности.

Лемма 5. Множество $\Omega_{T}$ замкнуто относительно равномерной сходимосmu $x(t) u$ *-слабой сходимости $u(t): \operatorname{ecлu}\left(x_{k}, u_{k}\right) \in \Omega_{T}, x_{k} \rightrightarrows x_{0}, u_{k} \stackrel{\text { *-сл. }}{\longrightarrow} u_{0}$, $m o\left(x_{0}, u_{0}\right) \in \Omega_{T}$.

ДокАЗАтЕЛьство. Замкнутость ограничений $x(0) \in M_{0}$ и $x(t) \in S(t)$ очевидно вытекает из замкнутости множества $M_{0}$ и множества $S(t)$ при всех $t \geqslant 0$. Замкнутость ограничения $\dot{x}=f(t, x, u)$ также очевидно вытекает из линейности $f$ по $u$, если перейти к интегральной форме этого уравнения.

Основная трудность - установить замкнутость включения $u(t) \in U(t, x(t))$. Это нетривиальньй факт, представляюший самостоятельньй интерес. Чтобы не отвлекаться далеко в сторону, мы здесь не приводим его доказательства. Отметим лишь, что он вытекает из замкнутости графика отображения $U(t, x)$ и выпуклости его значений (подробнее см., например, $[10, \S 8.5, \S 10.6])$. Лемма доказана.

Суммируя полученные факты, приходим к следующему утверждению.

Лемма 6. Множество $\Omega_{T}$ есть метризуемый компакт в пространстве пар $(x, u) \in A C[0, T] \times L_{\infty}[0, T]$ относительно произведения топологии, порохденной нормой $\|x\|_{C}, u *$-слабой топологии по $и$.

Обратимся теперь к функционалу $J_{T}$, который можно считать заданным на пространстве $C[0, T] \times L_{\infty}[0, T]$.

ЛЕмма 7. Пусть функиия ч удовлетворяет предполохсению П7 и даны последовательности $x_{k} \rightrightarrows x_{0}$ (равномерно $), u_{k} \stackrel{\text { *-сл. }}{\longrightarrow} u_{0}\left(\right.$ (слабо в $L_{\infty}$ относительно $\left.L_{1}\right)$ на отрезке $[0, T]$. Тогда

$$
\int_{0}^{T} \varphi\left(t, x_{0}(t), u_{0}(t)\right) d t \leqslant \liminf _{k \rightarrow \infty} \int_{0}^{T} \varphi\left(t, x_{k}(t), u_{k}(t)\right) d t
$$

т.е. функиионал $\int_{0}^{T} \varphi(t, x, u) d t$ полунепрерывен снизу относительно указанной сходимости.

Этот факт широко известен (см., например, [9], [10], [12], [13]). Отсюда и из непрерьвности $\beta(x)$ следует, что и наш функционал $J_{T}$ также полунепрерьвен снизу относительно указанной сходимости. 
4. Доказательство основной теоремы. Вернемся к рассмотрению задачи на бесконечном интервале. Введем сходимость в пространствах функций на $[0, \infty)$.

Пусть $C[0, \infty)$ есть пространство всех непрерьвных $n$-мерных функций на $[0, \infty)$. Для элементов этого пространства будем писать $x_{k} \rightrightarrows x_{0}$, если $x_{k}$ равномерно сходится к $x_{0}$ на любом отрезке $[0, T]$.

Для элементов введенного вьше пространства $L_{\infty}[0, \infty)$ будем писать $u_{k} \stackrel{\text { *-сл. }}{\longrightarrow} u_{0}$, если $u_{k} *$-слабо сходится к $u_{0}$ на любом отрезке $[0, T]$.

Допустимое множество $\Omega$ можно рассматривать как подмножество пространства $C[0, \infty) \times L_{\infty}[0, \infty)$. Как следует из его определения, $\Omega$ состоит из всех тех пар $(x, u)$, сужение которых на любой отрезок $[0, T]$ принадлежит соответствующему множеству $\Omega_{T}$.

Лемма 8. Допустимое множество $\Omega$ замкнуто относительно введенной сходимости, т.е. если $\left(x_{k}, u_{k}\right) \in \Omega, x_{k} \rightrightarrows x_{0}, u_{k} \stackrel{* \text {-сл. }}{\longrightarrow} u_{0}$, mo $\left(x_{0}, u_{0}\right) \in \Omega$.

ДокАЗАТЕЛЬСтво. По определению для любого $T>0$ имеем $\left(x_{k}, u_{k}\right) \in \Omega_{T}$ и $x_{k} \rightrightarrows x_{0}, u_{k} \stackrel{\text { *-сл. }}{\rightarrow} u_{0}$ на отрезке $[0, T]$, а так как по лемме 5 множество $\Omega_{T}$ замкнуто, то $\left(x_{0}, u_{0}\right) \in \Omega_{T}$. Отсюда и на всей полупрямой $\left(x_{0}, u_{0}\right) \in \Omega$, что и требовалось доказать.

Введем еще одно определение, которое будет удобно использовать в дальнейшем. На-

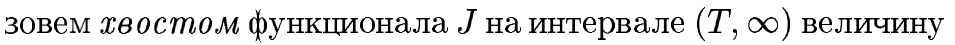

$$
\Theta_{T}(x, u)=\int_{T}^{\infty} \varphi(t, x(t), u(t)) d t .
$$

Это определение корректно, так как при вьполнении предположения П8 интеграл сходится (в расширенном смысле) на любой паре $(x, u) \in \Omega$.

ЛЕмма 9. Если на $\Omega$ выполняется предположсние П8, то отрицательные части хвостов функиионала стремятся $\kappa 0$ при $T \rightarrow \infty$ равномерно по всем $(x, u) \in \Omega$.

Более того, при той же функиии $\alpha(T)$ и при том же $T_{0}$ (фигурируюших в П8) для всех $(x, u) \in \Omega$ и всех $T \geqslant T_{0}$ выполнена оценка

$$
\Theta_{T}(x, u) \geqslant-\alpha(T) .
$$

ДокАЗАТЕЛЬСтво. Пусть выполняется предположение П8, т.е. найдутся $T_{0}$ и $\alpha(T) \rightarrow 0+$ такие, что для всех $T>T_{0}$ и всех $T^{\prime \prime}>T^{\prime} \geqslant T$ для любой пары $(x, u) \in \Omega$ выполнена оценка

$$
\int_{T^{\prime}}^{T^{\prime \prime}} \varphi(t, x(t), u(t)) d t \geqslant-\alpha(T) .
$$

Фиксируя здесь $T^{\prime}$ и устремляя $T^{\prime \prime} \rightarrow+\infty$, получаем требуемое неравенство (9).

Установим теперь аналог леммы 7 о полунепрерывности снизу функционала для бесконечного интервала.

ЛЕмма 10. Пусть выполнены предположения П1-П8. Тогда функиионал $J$ полунепрерывен снизу на $\Omega$ относительно введенной сходимости, т.е. если $\left(x_{k}, u_{k}\right) \in \Omega, x_{k} \rightrightarrows x_{0}, u_{k} \stackrel{\text { *-сл. }}{\longrightarrow} u_{0}$, mo

$$
J\left(x_{0}, u_{0}\right) \leqslant \liminf _{k \rightarrow \infty} J\left(x_{k}, u_{k}\right) .
$$


ДокАЗАТЕЛьСтво. Во-первых, по лемме 8 имеем $\left(x_{0}, u_{0}\right) \in \Omega$. Далее, пусть

$$
\liminf _{k \rightarrow \infty} J\left(x_{k}, u_{k}\right)=\mu_{0}
$$

Надо показать, что $J\left(x_{0}, u_{0}\right) \leqslant \mu_{0}$.

Опять считаем $\beta(\cdot) \equiv 0$. Обозначим для краткости $\varphi_{k}(t)=\varphi\left(t, x_{k}(t), u_{k}(t)\right)$. Для любого $T$ функционал $J\left(x_{k}, u_{k}\right)$ можно представить в виде

$$
J\left(x_{k}, u_{k}\right)=\int_{0}^{T} \varphi_{k}(t) d t+\Theta_{T}\left(x_{k}, u_{k}\right)
$$

Так как $J$ удовлетворяет предположению П8, то по лемме 9 для любого $T \geqslant T_{0}$ имеем $\Theta_{T} \geqslant-\alpha(T)$, поэтому

$$
\int_{0}^{T} \varphi_{k}(t) d t=J\left(x_{k}, u_{k}\right)-\Theta_{T}\left(x_{k}, u_{k}\right) \leqslant J\left(x_{k}, u_{k}\right)+\alpha(T),
$$

и, следовательно, при любом фиксированном $T$

$$
\liminf _{k \rightarrow \infty} \int_{0}^{T} \varphi_{k}(t) d t \leqslant \liminf _{k \rightarrow \infty} J\left(x_{k}, u_{k}\right)+\alpha(T)=\mu_{0}+\alpha(T) .
$$

Отсюда по лемме 7 получаем

$$
\int_{0}^{T} \varphi_{0}(t) d t \leqslant \mu_{0}+\alpha(T)
$$

Устремив теперь $T \rightarrow \infty$ и учитывая лемму 2 , получаем окончательно

$$
J\left(x_{0}, u_{0}\right)=\lim _{T \rightarrow \infty} \int_{0}^{T} \varphi_{0}(t) d t \leqslant \mu_{0} .
$$

Лемма доказана.

Следующий пример показьвает, что предположение П8 в этой лемме существенно: если его отбросить, она перестает быть верной.

ПРимеР. Рассмотрим последовательность функций $u_{k}(t)$, равных -1 при $t \in[k, k+1]$ и 0 в остальных случаях. Эта последовательность $*$-слабо сходится в нашем смысле к $u_{0} \equiv 0$. Положим $\varphi(t, x, u)=u, \beta(x)=0, f(t, x, u)=0, x(0)=0, U(t, x)=[-1,0]$.

Тогда все условия леммы 10 , кроме предположения П8, выполнены. Но при этом неравенство

$$
\int_{0}^{\infty} \varphi\left(u_{0}(t)\right) d t \leqslant \liminf _{k \rightarrow \infty} \int_{0}^{\infty} \varphi\left(u_{k}(t)\right) d t
$$

не вьполняется: слева имеем 0, а интеграл справа при всех $k$ есть

$$
\int_{0}^{\infty} \varphi\left(u_{k}(t)\right) d t=\int_{k}^{k+1}(-1) d t=-1 .
$$


ДОКАЗАТЕЛЬСТВО ОСНОВНОЙ ТЕОРЕМЫ. ВоЗьмем произвольную минимизирующую последовательность $\left(x_{k}, u_{k}\right) \in \Omega: J\left(x_{k}, u_{k}\right) \rightarrow \inf J=J_{*}$. Надо показать, что существует пара $(x, u) \in \Omega$, для которой $J(x, u)=J_{*}$.

1) Возьмем произвольное $T_{1}>0$. По лемме 6 множество $\Omega_{T_{1}}$ есть метризуемьй компакт. Поэтому из последовательности $\left(x_{k}, u_{k}\right)$ можно выбрать подпоследовательность $\left(x_{k}^{1}, u_{k}^{1}\right)$, сходящуюся на $\left[0, T_{1}\right]$ к некоторой паре $\left(x_{0}^{1}, u_{0}^{1}\right) \in \Omega_{T_{1}}$. (Говоря, что $x_{k}^{1}$ есть подпоследовательность последовательности $x_{k}$, мы имеем в виду, что существует возрастающая последовательность номеров $n_{k} \rightarrow \infty$ такая, что для любого $k x_{k}^{1}=x_{n_{k}}$.)

2) Возьмем любое $T_{2}>T_{1}+1$. По аналогии с предыдущим, из последовательности $\left(x_{k}^{1}, u_{k}^{1}\right)$ можно выбрать подпоследовательность $\left(x_{k}^{2}, u_{k}^{2}\right)$, сходящуюся на $\left[0, T_{2}\right]$ к некоторой паре $\left(x_{0}^{2}, u_{0}^{2}\right) \in \Omega_{T_{2}}$.

При этом, так как $\left(x_{k}^{2}, u_{k}^{2}\right)$ - подпоследовательность последовательности $\left(x_{k}^{1}, u_{k}^{1}\right)$, то на $\left[0, T_{1}\right]$ их пределы совпадают: $x_{0}^{2} \equiv x_{0}^{1}, u_{0}^{2} \equiv u_{0}^{1}$, т.е. новая предельная пара есть продолжение старой на отрезок $\left[T_{1}, T_{2}\right]$.

3) Далее возьмем любое $T_{3}>T_{2}+1$ и из последовательности $\left(x_{k}^{2}, u_{k}^{2}\right)$ выберем подпоследовательность $\left(x_{k}^{3}, u_{k}^{3}\right)$, и т.д.

Таким образом, на $m$-ом шаге данной процедуры мы имеем $T_{m}>T_{m-1}+1$ и последовательность $\left(x_{k}^{m}, u_{k}^{m}\right)$, сходящуюся к некоторой паре $\left(x_{0}^{m}, u_{0}^{m}\right) \in \Omega_{T_{m}}$, которая на предыдущем отрезке $\left[0, T_{m-1}\right]$ совпадает с $\left(x_{0}^{m-1}, u_{0}^{m-1}\right)$.

Определим пару $\left(x_{0}, u_{0}\right) \in A C \times L_{\infty}[0, \infty)$, которая на каждом отрезке $\left[0, T_{m}\right]$ совпадает с $\left(x_{0}^{m}, u_{0}^{m}\right)$. Такое определение корректно, ибо при любом $k>m$ на отрезке $\left[0, T_{m}\right]$ имеем совпадение $\left(x_{0}^{k}, u_{0}^{k}\right) \equiv\left(x_{0}^{m}, u_{0}^{m}\right)$. Тогда на каждом $\left[0, T_{m}\right]$ имеем $\left(x_{0}, u_{0}\right) \in \Omega_{T_{m}}$, откуда по определению $\Omega$ вытекает, что на всей полупрямой $\left(x_{0}, u_{0}\right) \in \Omega$.

4) Рассмотрим диагональную последовательность $\left(x_{k}^{k}, u_{k}^{k}\right)$. Она, очевидно, обладает следуюшим свойством: для любого фиксированного $m$ при всех $k \geqslant m$ эта последовательность содержится в последовательности $\left(x_{i}^{m}, u_{i}^{m}\right), i=1,2, \ldots$, и поэтому на отрезке $\left[0, T_{m}\right]$ она сходится к $\left(x_{0}^{m}, u_{0}^{m}\right)=\left(x_{0}, u_{0}\right)$. А поскольку $T_{m} \rightarrow \infty$, последовательность $\left(x_{k}^{k}, u_{k}^{k}\right)$ сходится к $\left(x_{0}, u_{0}\right)$ на любом отрезке $[0, T]$, т.е. по определению сходится в пространстве $C[0, \infty) \times L_{\infty}[0, \infty)$.

5) Рассмотрим теперь функционал $J\left(x_{k}^{k}, u_{k}^{k}\right)$. По лемме 10 он полунепрерывен снизу относительно данной сходимости, поэтому

$$
J\left(x_{0}, u_{0}\right) \leqslant \liminf _{k \rightarrow \infty} J\left(x_{k}^{k}, u_{k}^{k}\right)=J_{*}
$$

и так как $J_{*}=\inf J$, тонеравенство $J\left(x_{0}, u_{0}\right)<J_{*}$ невозможно, и значит, $J\left(x_{0}, u_{0}\right)=J_{*}$, что и требовалось доказать.

ЗАмечАниЕ. Так как для всех $T>0$ сходимость в $\Omega_{T}$ задается некоторой метрикой $\rho_{T}$, то сходимость в $\Omega$ также может быть задана метрикой, в качестве которой можно взять, например, стандартную комбинацию

$$
\rho\left(\left(x^{\prime}, u^{\prime}\right),\left(x^{\prime \prime}, u^{\prime \prime}\right)\right)=\sum_{m=1}^{\infty} \frac{1}{2^{m}} F\left(\rho_{T_{m}}\left(\left(x^{\prime}, u^{\prime}\right),\left(x^{\prime \prime}, u^{\prime \prime}\right)\right)\right)
$$

где $T_{m} \rightarrow \infty$, а $F(z)=z /(1+z)$. В пунктах 1$\left.)-4\right)$ приведенного доказательства фактически установлено, что из каждой последовательности элементов $\Omega$ можно выбрать 
сходящуюся подпоследовательность, т.е. что в этой метрике $\Omega$ есть компакт. Таким образом, наша основная теорема полностью укладывается в рамки общего принципа Вейерштрасса: полунепрерывная снизу функция на компакте достигает своей нижней грани.

5. Частные случаи. Приведем некоторые условия, гарантирующие выполнение предположения П8.

1. Наиболее простое условие, которое отмечается во многих работах и которое действительно наиболее часто выполнено в конкретных задачах, состоит в том, что $\varphi(t, x, u)$ ограничена снизу интегрируемой функцией, т.е. существует такая $l(t) \in L_{1}(0, \infty), l(t) \geqslant 0$, что

$$
\varphi(t, x(t), u(t)) \geqslant-l(t) \quad \text { п.в. на }(0, \infty)
$$

для любой пары $(x, u) \in \Omega$. В этом случае имеем очевидную оценку для кусков функционала

$$
\int_{T^{\prime}}^{T^{\prime \prime}} \varphi(t, x(t), u(t)) d t \geqslant \int_{T^{\prime}}^{T^{\prime \prime}}-l(t) d t \geqslant \int_{T^{\prime}}^{\infty}-l(t) d t=-\alpha\left(T^{\prime}\right),
$$

и так как $\alpha\left(T^{\prime}\right) \rightarrow 0$ при $T^{\prime} \rightarrow \infty$, то наше условие $(6)$ выполнено.

2. Пусть все ограничения задачи не зависят от $t$, а функционал имеет вид

$$
J=\beta(x(0))+\int_{0}^{\infty} e^{-r t} L(x, u) d t \rightarrow \max
$$

где функция $L$ непрерывна по $(x, u)$ и вогнута (т.е. вьпукла вверх) по $u$; a $r$ - некоторое положительное число (оно называется показателем дисконтирования). Такие задачи характерны для динамических моделей математической экономики [1]-[7].

Предположим, что существуют такие неотрицательные числа $p, h, q, C, K$, что для всех $x \in S, u \in U(x)$ вьполняются оценки

$$
\begin{gathered}
(x, f(x, u)) \leqslant p\left(|x|^{2}+h\right), \\
L(x, u) \leqslant C|x|^{q}+K,
\end{gathered}
$$

и пусть $p q<r$. Тогда функция $\varphi(t, x, u)=e^{-r t} L(x, u)$ ограничена сверху интегрируемой функцией, и, следовательно, согласно п. 1 для задачи максимизации предположение П8 вьполнено.

Действительно, рассмотрим функцию $z(t)=|x(t)|^{2}+h$. Из (11) следует, что $|\dot{z}| \leqslant 2 p z$, откуда $z(t) \leqslant z(0) e^{2 p t}$ и поэтому $|x(t)| \leqslant C_{1} e^{p t}$ с некоторой константой $C_{1}$. Тогда $L(x, u) \leqslant C_{2} e^{p q t}+K$ с некоторой $C_{2}$, и отсюда

$$
e^{-r t} L(x, u) \leqslant C_{2} e^{(p q-r) t}+K e^{-r t}=l(t)
$$

где функция $l(t)$ интегрируема на $(0, \infty)$, поскольку $p q-r<0$. 
3. Рассмотрим также случай, когда задача имеет тот же вид, что и в п. 2 , но управляемая система линейна (с постоянньпи коэффициентами): $\dot{x}=A x+B u$, а множество управлений $U$ не зависит от $x$. Пусть число $p$ таково, что все собственные значения $\lambda$ матрицы $A$ имеют $\operatorname{Re} \lambda<p$, и существуют такие неотрицательные числа $q, C, K$, что для всех $x \in S, u \in U$ вьполняется оценка (12) и при этом $p q<r$. Тогда опять семейство функций $\varphi(t, x, u)=e^{-r t} L(x, u)$ ограничено сверху интегрируемой функцией, и, следовательно, предположение П8 вьполнено.

Действительно, в этом случае, как известно, найдется такая константа $C_{1}$, что любое решение системы $\dot{x}=A x+B u, u \in U$, с начальньм значением $x(0) \in M_{0}$ удовлетворяет оценке $|x(t)| \leqslant C_{1} e^{p t}$, и далее надо повторить соответствующие рассуждения предыдущего пункта.

6. Сравнение с известными результатами. Наиболее общие результаты по данному вопросу были получены в работе Балдера [3]. Сравним его и наше предположения относительно поведения функционала на бесконечности. В указанной работе введено понятие сильной равномерной интегрируемости семейства функций и потребовано, чтобы семейство $\left\{\varphi^{-}(t, x(t), u(t))\right\}$ обладало этим свойством.

Пусть $M$ - измеримое множество на прямой. Через $L_{1}(M)$ обозначим пространство измеримых интегрируемых по Лебегу функций, а через $L_{1}^{+}(M)$ - множество всех неотрицательных функций из $L_{1}(M)$.

Напомним, что множество функций $G \subset L_{1}(M)$ называется равномерно интегриpуемым (на $M$ ), если для любого $\varepsilon>0$ существует такое $\delta>0$, что если измеримое множество $E \subset M$ имеет mes $E<\delta$, то

$$
\int_{E}|g(t)| d t<\varepsilon
$$

для всех $g \in G$.

ОПРЕДЕЛЕНИЕ 1 (Балдер [3]). Множество функций $G \subset L_{1}(M)$ называется сильно равномерно интегрируемым, если для любого $\varepsilon>0$ существует $h \in L_{1}^{+}(M)$ такая, что

$$
\int_{E(|g|>h)}|g(t)| d t<\varepsilon \quad \text { для любой } g \in G,
$$

где $E(f>h)=\{t \in M \mid f(t)>h(t)\}$.

Нетрудно заметить, что для множества $M$ конечной меры это свойство совпадает с равномерной интегрируемостью, а для множества бесконечной меры оно сильнее последнего свойства.

Вместо определения 1 мы будем пользоваться следуюшим эквивалентным определением 2 , которое представляется нам более удобным.

Пусть $h, g \in L_{1}^{+}(M)$. Будем говорить, что $h$ мажсорирует $g$, если $h(t) \geqslant g(t)$ п.в. на $M$, и $h$ мажсорирует $g$ с интегральной точностью $\varepsilon>0$, если

$$
\int_{M}(g(t)-h(t))^{+} d t<\varepsilon
$$


ОПРЕДЕЛЕНИЕ 2. Множество функций $G \subset L_{1}(M)$ назовем сильно равномерно интегрируемым, если для любого $\varepsilon>0$ существует такая функция $h \in L_{1}^{+}(M)$, которая мажорирует $|g(t)|$ с интегральной точностью $\varepsilon$ для любой $g \in G$.

ЛЕмма 11. Определение 1 и определение 2 әквивалентны.

ДокАЗАТЕЛЬСтво. Без нарушения общности считаем, что $G \subset L_{1}^{+}(M)$. Так как $h \geqslant 0$, то всегда $g-h \leqslant g$, следовательно,

$$
\int_{M}(g-h)^{+} d t=\int_{E(g>h)}(g-h) d t \leqslant \int_{E(g>h)} g d t
$$

поэтому любое множество $G$, удовлетворяющее определению 1 , удовлетворяет и определению 2.

Установим обратное. Пусть множество $G$ удовлетворяет определению 2. Допустим, что оно не удовлетворяет определению 1 , т.е. существует $\varepsilon>0$ такое, что для любого $h \in L_{1}^{+}(M)$ найдется $g_{h} \in G$, для которой

$$
\int_{E_{h}} g_{h} d t \geqslant \varepsilon>0, \quad \text { где } E_{h}=E\left(g_{h}>h\right) .
$$

Фиксируем это $\varepsilon$. По определению 2 для него существует $h_{0} \in L_{1}^{+}(M)$ такое, что для любого $g \in G$ выполнено

$$
\int_{M}\left(g-h_{0}\right)^{+} d t<\frac{\varepsilon}{2}
$$

Тогда и для всех $h \geqslant h_{0}$ выполнено

$$
\int_{M}(g-h)^{+} d t<\frac{\varepsilon}{2}
$$

Поэтому для любого $h \geqslant h_{0}$ имеем

$$
\int_{M}\left(g_{h}-h\right)^{+} d t=\int_{E_{h}}\left(g_{h}-h\right) d t<\frac{\varepsilon}{2},
$$

и отсюда с учетом (13) получаем

$$
\int_{E_{h}} h d t>\frac{\varepsilon}{2} .
$$

По определению на $E_{h}$ всегда имеем $g_{h}-h / 2=\left(g_{h}-h\right)+h / 2>h / 2$, откуда с учетом предыдущего неравенства получаем

$$
\int_{E_{h}}\left(g_{h}-\frac{h}{2}\right) d t>\frac{\varepsilon}{4}
$$

и так как $E\left(g_{h}>h / 2\right) \supset E\left(g_{h}>h\right)=E_{h}$, то

$$
\int_{E\left(g_{h}>h / 2\right)}\left(g_{h}-\frac{h}{2}\right) d t=\int_{M}\left(g_{h}-\frac{h}{2}\right)^{+} d t>\frac{\varepsilon}{4} .
$$


Полученное неравенство выполнено для любого $h \geqslant h_{0}$. Но это противоречит определению 2 , согласно которому найдется $h \geqslant h_{0}$ такое, что для любого $g \in G$

$$
\int_{M}\left(g-\frac{h}{2}\right)^{+} d t<\frac{\varepsilon}{4}
$$

Лемма доказана.

Рассмотрим случай $M=(0,+\infty)$. Пусть множество $\Phi \subset L_{1}(0, \infty)$ таково, что семейство функций $\left\{\varphi^{-} \mid \varphi \in \Phi\right\}$ сильно равномерно интегрируемо. Пользуясь определением 2 и равенством $a^{-}=(-a)^{+}$, нетрудно показать, что это условие эквивалентно следуюшему: для любого $\varepsilon>0$ найдется $h_{\varepsilon} \in L_{1}^{+}(0, \infty)$ такая, что для всех $\varphi \in \Phi$

$$
\int_{0}^{\infty}\left(\varphi+h_{\varepsilon}\right)^{-} d t<\varepsilon
$$

Покажем, что в этом случае и наше условие о равномерной сходимости отрицательной части кусков функционала к нулю для множества $\Phi$ выполнено. Имеем при любых $T^{\prime}<T^{\prime \prime}$ следующую оценку:

$$
\int_{T^{\prime}}^{T^{\prime \prime}}\left(\varphi+h_{\varepsilon}\right)^{-} d t \leqslant \int_{0}^{\infty}\left(\varphi+h_{\varepsilon}\right)^{-} d t<\varepsilon
$$

В силу интегрируемости $h_{\varepsilon}$ найдется $T_{\varepsilon}$ такое, что

$$
\int_{T_{\varepsilon}}^{\infty} h_{\varepsilon} d t<\varepsilon
$$

Представим $\varphi$ в виде $\varphi=\left(\varphi+h_{\varepsilon}\right)+\left(-h_{\varepsilon}\right)$. Так как функция $(\cdot)^{-}$сублинейна, а $h_{\varepsilon} \geqslant 0, \operatorname{\operatorname {~}о} \varphi^{-} \leqslant\left(\varphi+h_{\varepsilon}\right)^{-}+\left(-h_{\varepsilon}\right)^{-}=\left(\varphi+h_{\varepsilon}\right)^{-}+h_{\varepsilon}$.

Тогда при любых $T^{\prime \prime}>T^{\prime}>T_{\varepsilon}$ имеем

$$
\int_{T^{\prime}}^{T^{\prime \prime}} \varphi^{-} d t \leqslant \int_{T^{\prime}}^{T^{\prime \prime}}\left(\varphi+h_{\varepsilon}\right)^{-} d t+\int_{T^{\prime}}^{T^{\prime \prime}} h_{\varepsilon} d t<\varepsilon+\varepsilon=2 \varepsilon
$$

и отсюда опять же в силу сублинейности функции $(\cdot)^{-}$

$$
\left(\int_{T^{\prime}}^{T^{\prime \prime}} \varphi d t\right)^{-} \leqslant \int_{T^{\prime}}^{T^{\prime \prime}} \varphi^{-} d t<2 \varepsilon
$$

следовательно, предположение П8 для множества $\Phi$ также вьполнено.

Однако, обратное утверждение, очевидно, неверно уже потому, что функции, удовлетворяюшие $\Pi 8$, не обязательно интегрируемы по Лебегу на $(0, \infty)$ (т.е. их интегралы не обязательно сходятся абсолютно). Например, если вместо функций $\varphi(t) \in \Phi$ рассмотреть $\varphi(t)+\frac{1}{t+1} \sin t$, то полученные функции уже не будут принадлежать $L_{1}(0, \infty)$, тогда как куски их интегралов по-прежнему будут удовлетворять оценке (6), поскольку

$$
\int_{T^{\prime}}^{T^{\prime \prime}} \frac{\sin t}{t+1} d t=-\left.\frac{\cos t}{t+1}\right|_{T^{\prime}} ^{T^{\prime \prime}}-\int_{T^{\prime}}^{T^{\prime \prime}} \frac{\cos t}{(t+1)^{2}} d t \rightarrow 0
$$

при $T^{\prime}, T^{\prime \prime} \rightarrow \infty$. Таким образом, требование сильной равномерной интегрируемости семейства функций, принятое в работе [3], более жесткое, чем наше требование П8.

Если даже ослабить условие работы [3], потребовав сильной равномерной интегрируемости семейства функций $\left\{\varphi^{-}(t)\right\}$ лишь на интервале $(T, \infty)$ при достаточно большом $T$, то в силу тех же причин это ослабленное требование останется строго сильнее нашего условия П8. 
7. Возможные обобщения. 1. Если в задаче (1)-(5) присутствуют дополнительные ограничения неравенства вида

$$
J_{i}=\beta_{i}(x(0))+\int_{0}^{\infty} \varphi_{i}(t, x, u) d t \leqslant 0, \quad i=1, \ldots, m,
$$

где функции $\beta_{i}, \varphi_{i}$ удовлетворяют тем же предположениям, что и $\beta, \varphi$ (в том числе и предположению П8), а интеграл по $[0, \infty)$ по-прежнему понимается как предел интегралов по отрезкам $[0, T]$ при $T \rightarrow \infty$, то ясно, что основная теорема останется верной и доказательство не изменится. Действительно, функционалы $J_{j}$ будут полунепрерьвны снизу на “старом" множестве $\Omega$ (не учитьвающем ограничение (14)) относительно введенной сходимости, поэтому множество пар $(x, u) \in \Omega$, удовлетворяющих ограничению (14), будет замкнутьм, а тогда новое множество допустимых траекторий, задающееся теперь ограничениями (2)-(5) и (14), по-прежнему будет компактным.

2. Можно также добавить в задачу и ограничения равенства вида

$$
F_{j}=\beta_{j}(x(0))+\int_{0}^{\infty} f_{j}(t, x, u) d t=0, \quad j=1, \ldots, k,
$$

где функции $f_{j}$ удовлетворяют предположению П1 (т.е. они непрерьвны по $(t, x)$ и линейны по $u$ ), а $\beta_{j}$ - предположению П6 (т.е. $\beta_{j}(x)$ непрерьвны). При этом мы потребуем, чтобы функции $f_{j}$ и $-f_{j}$ удовлетворяли предположению П8, т.е. чтобы

$$
\int_{T^{\prime}}^{T^{\prime \prime}} f_{j}(t, x(t), u(t)) d t \rightarrow 0 \quad \text { при } \quad T^{\prime}, T^{\prime \prime} \rightarrow \infty
$$

равномерно по “старому" множеству $\Omega$. Последнее условие эквивалентно тому, что для любой допустимой пары интегралы в (15) имеют конечные значения, причем они сходятся к своим значениям равномерно по всем допустимым траекториям. В этой ситуации опять основная теорема останется справедливой, так как множество пар $(x, u) \in \Omega$, удовлетворяющих (15), замкнуто относительно введенной сходимости.

Ограничение (15) позволяет рассматривать финальные ограничения равенства на конец траектории

$$
x(\infty)=\lim _{T \rightarrow \infty} x(T)
$$

типа

$$
\left(a_{j}, x(\infty)\right)=c_{j}, \quad j=1, \ldots, k,
$$

где $a_{j} \in \mathbb{R}^{n}$, которые в эквивалентной форме могут быть представлены в виде

$$
\left(a_{j}, x(0)\right)+\int_{0}^{\infty}\left(a_{j}, f(t, x(t), u(t))\right) d t=c_{j}, \quad j=1, \ldots, k .
$$

Здесь надо предполагать, что функции $\pm\left(a_{j}, f(t, x, u)\right)$ удовлетворяют П8.

3. Задача, в которой вместо управляемой системы имеется дифференциальное включение $\dot{x} \in V(t, x)$, где многозначное отображение $V$ удовлетворяет предположению П4, легко сводится к рассмотренной. Для этого надо перейти к системе $\dot{x}=u, u \in V(t, x)$. 
4. В настоящей статье мы предполагали, что система (2) линейна по управлению, а множество $U(t, x)$ есть вьпуклый компакт. В случае общей нелинейной системы надо требовать, чтобы выпуклым и компактным было так назьваемое расииренное множество скоростей [10], [3]:

$$
Q(t, x)=\left\{(z, v) \in \mathbb{R}^{1+n} \mid \exists u \in U(t, x): z \geqslant \varphi(t, x, u), v=f(t, x, u)\right\},
$$

при этом надо рассматривать сходимость траекторий $x(t)$ в пространстве $C[0, T]$, a для представления предельной траектории в виде решения системы $(2)$ при некотором управлении $u(t)$ применять один из вариантов теоремы об измеримом выборе, например, лемму Филиппова о включении [8].

Мы не стали рассматривать этот общий случай, так как соответствующие технические усложнения касаются задачи на фиксированном отрезке (и они хорошо известны), а нашей целью было как можно более четко выделить специфику задачи на бесконечном интервале. Отметим лишь, что этот общий случай укладывается в предлагаемую ниже абстрактную схему.

8. Абстрактная схема. Изложенная выше схема доказательствапроходит и в следующей абстрактной постановке.

Пусть имеется возрастающее счетное семейство множеств $T_{n} \subset T_{n+1} \subset \cdots, n=$ $1,2, \ldots$, и пусть $\Re=\cup T_{n}$. На каждом $T_{n}$ задано некотороемножество функций $\Omega\left(T_{n}\right)=$ $\left\{w: T_{n} \rightarrow Z\right\}$, принимающих значения в некотором множестве образов $Z$, и задан функционал $J_{n}: \Omega\left(T_{n}\right) \rightarrow \mathbb{R}$. Предполагается, что для любого $n$ выполнено $\left.\Omega\left(T_{n+1}\right)\right|_{T_{n}} \subset$ $\Omega\left(T_{n}\right)$.

Пусть $\Omega$ есть множество всех функций $w: \Re \rightarrow Z$ таких, что для любого $n$ сужение $w_{n}=\left.w\right|_{T_{n}}$ принадлежит $\Omega\left(T_{n}\right)$. Тогда для каждой функции $w \in \Omega$ определены функционалы $J_{n}(w)=J_{n}\left(w_{n}\right), n=1,2, \ldots$, и поэтому можно рассматривать функционал

$$
J(w)=\lim _{n \rightarrow \infty} J_{n}\left(w_{n}\right) .
$$

Точнее, он рассматривается лишь на множестве тех $w \in \Omega$, для которых этот предел (конечный или бесконечньй) существует.

Ставится задача: $J(w) \rightarrow \min$ по всем $w \in \Omega$, для которых функционал существует.

ПРЕДПоложЕНИЯ. S1. Для каждого $n$ множество функций $\Omega\left(T_{n}\right)$ есть компакт относительно некоторой топологии $\tau_{n}$, имеющей счетную базу (и значит, метризуемой); при этом отображение $\pi_{n}: \Omega\left(T_{n+1}\right) \rightarrow \Omega\left(T_{n}\right)$, ставящее в соответствие каждой функции $w(t)$ на множестве $T_{n+1}$ ее сужение на $T_{n}$, непрерьвно.

S2. Для каждого $n$ функционал $J_{n}$ полунепрерьвен снизу на $\Omega\left(T_{n}\right)$ относительно топологии $\tau_{n}$.

S3. Существуют числовая последовательность $\alpha_{N} \rightarrow 0+$ и номер $N_{0}$ такие, что для любого $N>N_{0}$ и для любых $n_{2}>n_{1} \geqslant N$, для любого $w \in \Omega$

$$
J_{n_{2}}(w)-J_{n_{1}}(w) \geqslant-\alpha_{N}
$$

или, что то же самое,

$$
\left(J_{n_{2}}(w)-J_{n_{1}}(w)\right)^{-} \rightarrow 0 \quad \text { при } n_{1}, n_{2} \rightarrow \infty, n_{1}<n_{2},
$$


равномерно по всем $w \in \Omega$.

Таким образом, в этой абстрактной схеме роль кусков функционала играют разности $J_{n_{2}}(w)-J_{n_{1}}(w)$.

При сделанных предположениях справедлив аналог леммы 2, гарантирующий существование предела функционала (как и ранњше, либо конечного, либо $+\infty$ ) на любой $w \in \Omega$, множество $\Omega$ есть метризуемьй компакт в топологии сходимости на каждом $T_{n}$, функционал $J$ полунепрерывен снизу на $\Omega$ относительно этой сходимости, и верна следующая

ТЕОрема 2. Пусть существует $w \in \Omega$, на которой $J(w)<+\infty$. Тогда поставленная задача имеет решение, т.е. J достигает своего минимума на $\Omega$.

ДокАЗАТЕЛЬСТВО повторяет основные пункты доказательства теоремы 1.

Можно предложить и еще более абстрактную схему, в которой множеств $T_{n}$ нет, а вместо пространств функций $\Omega\left(T_{n}\right)$ рассматривается произвольное проективное семейство компактов $\Omega_{n}$ со счетньми базами, и $\Omega$ есть проективньй предел этого семейства. Однако для такой постановки (и тем более при отказе от счетности баз у топологий $\tau_{n}$ ) пока нет достаточно убедительной мотивировки, поэтому мы здесь детально ее не рассматриваем.

Авторы выражают благодарность Б. А. Копейкину за полезные обсуждения.

\section{СПИСОК ЦИТИРОВАННОЙ ЛИТЕРАТУРЫ}

[1] Baum R. F. Existence theorems for Lagrange control problems with unbounded time domain // J. Optimization Theory Appl. 1976. V. 19. P. 89-116.

[2] Magill M. Infinite horizon programs // Econometrica. 1981. V. 49. P. 679-711.

[3] Balder E. J. An existence result for optimal economic growth problems // J. Math. Anal. Appl. 1983. V. 95. P. 195-213.

[4] Carlson D. A., Haurie A. B., Leizarowitz A. Infinite-Horizon Optimal Control. Berlin: Springer, 1991.

[5] Leonard D., Long N. V. Optimal Control Theory and Static Optimization in Economics. Cambridge: Cambridge Univ. Press, 1992.

[6] Zaslavski A.J. Optimal programs on infinite horizon // SIAM J. Control Optimization. 1995. V. 33. №6. P. 1643-1686.

[7] Zaslavski A. J. Turnpike property of optimal solutions of infinite-horizon variational problems // SIAM J. Control Optimization. 1997. V. 35. № 4. P. 1169-1203.

[8] Филиппов А.Ф. О некоторых вопросах теории оптимального регулирования // Вестн. МГУ. Сер. матем., мех., астрон., физ., хим. 1959. № 2. С. 25-32.

[9] Иоффе А. Д., Тихомиров В. М. Теория экстремальных задач. М.: Наука, 1974.

[10] Cesari L. Optimization: Theory and Applications. New York: Springer, 1983.

[11] Колмогоров А. Н., Фомин С. В. Элементы теории функций и функционального анализа. M.: Наука, 1968.

[12] Ioffe A. D. On lower semicontinuity of integral functions // SIAM J. Control Optimization. 1977. V. 15. P. 521-538.

[13] Olech C. Weak lower semicontinuity of integral functions // J. Optimization Theory Appl. 1976. V. 15. P. 3-16.

(А. В. Дмитрук) Центральный экономико-математический 